\title{
Identifying the affected hemisphere with a multimodal approach in MRI-positive or negative, unilateral or bilateral temporal lobe epilepsy
}

This article was published in the following Dove Press journal:

Neuropsychiatric Disease and Treatment

7 January 2014

Number of times this article has been viewed

\author{
Jing Zhang' \\ Qingzhu Liu \\ Shanshan $\mathrm{Mei}^{2}$ \\ Xiaoming Zhang ${ }^{2}$ \\ Weifang Liu' \\ Hui Chen' \\ Hong Xia' \\ Zhen Zhou' \\ Xiaofei Wang ${ }^{2}$ \\ Yunlin $\mathrm{Li}^{2}$ \\ 'School of Biomedical Engineering, \\ Capital Medical University, Beijing, \\ People's Republic of China; \\ ${ }^{2}$ Department of Functional Neurology \\ and Neurosurgery, Beijing Haidian \\ Hospital, Beijing, People's Republic of \\ China
}

Correspondence: Jing Zhang School of Biomedical Engineering, Capital Medical University, Beijing 100069, People's Republic of China Tel $+86108391 \quad 1543$

Fax +86 I0 839II544

Email jzhang0000@163.com
Abstract: Patients with non-lesional or bilateral temporal-lobe epilepsy (TLE) are often excluded from surgical treatment. This study investigated focus lateralization in TLE to understand identification of the affected hemisphere with regard to non-lesional or bilateral affection and postsurgical outcome. A total of 24 TLE patients underwent presurgical evaluation with magnetic resonance imaging (MRI), proton magnetic resonance spectroscopy ( $\left.{ }^{1} \mathrm{H}-\mathrm{MRS}\right)$, video-electroencephalogram (video-EEG), and/or intracranial EEG (icEEG), and they were classified as MRI-positive or negative, unilateral or bilateral TLE cases. In patients with positiveMRI, MRI and ${ }^{1} \mathrm{H}-\mathrm{MRS}$ indicated high (100\%) concordant lateralization to EEG findings in unilateral TLE, and moderate (75\%) concordance to icEEG findings in bilateral TLE; whereas in patients with negative-MRI, ${ }^{1} \mathrm{H}-\mathrm{MRS}$ indicated moderate $(60 \%-75 \%)$ concordance to EEG and/or icEEG in unilateral TLE, and relatively low (50\%) concordance to icEEG in bilateral TLE. Ninety point nine percent of patients with unilateral TLE and $41.7 \%$ of patients with bilateral TLE (including 50\% of MRI-negative bilateral TLE) became seizure-free. The MRS findings were not correlated with seizure outcome, while non-seizure-free patients had an insignificantly higher percentage of contralateral $\mathrm{N}$-acetyl aspartate (NAA) reduction compared with seizure-free patients, indicating the relatively low predictive value of ${ }^{1} \mathrm{H}-\mathrm{MRS}$ for surgical outcome. Further, EEG and icEEG findings were significantly correlated with seizure outcome, and for patients with positive MRI, MRI findings were also correlated with seizure outcome, indicating the predictive value of these modalities. The results suggested that a multimodal approach including neuroimaging, EEG, and/or icEEG could identify seizure focus in most cases, and provide surgical options for non-lesional or bilateral TLE patients with a possible good outcome.

Keywords: focus lateralization, MRS, neuroimaging, surgical outcome

\section{Introduction}

Around $30 \%$ of patients with partial seizures are resistant to antiepileptic drugs and may need surgical treatment. Temporal lobe epilepsy (TLE) is the most common type of epilepsy. Hippocampal sclerosis (HS) is the most common cause of TLE in adult patients, ${ }^{1,2}$ while malformations of cortical development (MCD) is more commonly seen in pediatric patients. ${ }^{3}$ Epilepsy surgery opens the possibility of complete seizure control and brings the hope of seizure-free outcome. Over decades, epilepsy surgery has improved gradually and approached 60\%-90\% seizure-free outcome in patients with TLE and 40\%-60\% in extratemporal lobe epilepsy (ETLE) ${ }^{4}$

Correct lateralization of the affected hemisphere with precise localization of the epileptic foci is a prerequisite for good surgical outcome, but it remains a challenge, 
especially for non-lesional epilepsy, bilateral TLE, and ETLE. Neuroimaging such as magnetic resonance imaging (MRI), magnetic resonance spectroscopy (MRS), and positronemission tomography (PET) can detect lesions and other structural or functional abnormalities, and is thus important in focus lateralization and localization. Concordance of different imaging modalities in localization of seizure focus increases the confidence of correct hemispheric lateralization in TLE. ${ }^{5-7}$ However, when the clinical, electrophysiological and neuroimaging findings are discordant or the focus lateralization and localization are unclear, intracranial electroencephalogram (icEEG) with deep electrodes is needed to further localize the seizure focus. ${ }^{6-8}$ IcEEG monitoring with surgically implanted electrodes is critical for the assessment of patients with drug-resistant epilepsy (especially in challenging cases) in presurgical evaluation. It has good sensitivity and spatial specificity, but limited spatial sampling, and requires additional surgical procedure, which increases patient discomfort, immobility, risks for complications, and medical costs. ${ }^{9}$ Neuroimaging is useful in planning icEEG implantation, and concordant neuroimaging findings may minimize the need for invasive icEEG. ${ }^{10}$

Nevertheless, patients with drug-resistant bilateral TLE (ie, seizures originating from both temporal lobes) are still often excluded from surgical treatment. ${ }^{11,12}$ This is because surgery is only performed in one hemisphere to avoid severe functional deficits (due to bilateral resection), and it is often difficult to identify the side of seizure origin (ie, the epileptic focus in one hemisphere that initiates most seizures and causes the most severe seizure). ${ }^{13}$ In addition, poor focus lateralization and/or localization often lead to unfavorable seizure outcome in bilateral TLE. ${ }^{14}$ Furthermore, apart from bilateral TLE, focus lateralization and localization in nonlesional TLE is also difficult due to the absence of imaging guidance for resection. Therefore, how to improve focus lateralization and localization in TLE (especially in challenging cases such as non-lesional or bilateral TLE) remains a challenge.

This study investigated presurgical focus lateralization in 24 TLE patients with neuroimaging (MRI and proton MRS [ $\left.{ }^{1} \mathrm{H}-\mathrm{MRS}\right]$ ), video-EEG, and/or icEEG. The patients were classified as MRI positive or negative, unilateral or bilateral TLE cases. Ictal video-EEG and/or icEEG findings were used as the "gold standard" for focus lateralization comparison, surgical outcomes were compared between different subgroups of patients, and the relationship between presurgical findings and seizure outcome was explored.

\section{Methods}

\section{Subjects}

A total of 24 consecutive patients with drug-resistant epilepsy who were admitted to the Department of Functional Neurology and Neurosurgery, Beijing Haidian Hospital during 2010 (July)-2012 (April) were included in this study. All patients were diagnosed as having TLE and underwent presurgical evaluation, and of these, 23 underwent surgery. Surgical outcomes were evaluated with Engel classification ${ }^{15}$ during patients' postoperative visits, and the patients' follow-up lasted for 1.5-3.0 years. This study was approved by the Institutional Review Board at the Capital Medical University.

\section{EEG recordings}

The simultaneous registration of continuous video-EEG monitoring was performed using the Nicolet system (Natus Medical Incorporated, San Carlos, CA, USA). Simultaneous documentation of behavior via a split-screen technique was used over a period of 4-264 hours (0.17-11.00 days) for all patients, and over 200 hours ( 8.3 days) for most patients. Technical details were: number of EEG/polygraphic channels, 32; sampling rate, $256 \mathrm{~Hz}$. EEG electrodes were placed according to the international 10/20 system of EEG. EEG analysis was performed using continuous 24-hour video-EEG monitoring for most patients. EEG analysis was performed without knowledge of imaging results. Interictal epileptiform discharges and ictal EEG findings were counted, and unilateral or bitemporal activity was reported by EEG specialists. Based on the frequency of unilateral or bitemporal EEG activity (together with icEEG findings if available), unilateral or bitemporal TLE was determined with the following rules. Briefly, if ictal EEG activity was bitemporal, then the patient was considered as having bitemporal TLE; if ictal EEG activity was unilateral, but interictal or ictal icEEG activity was bilateral, then the patient was still considered as having bilateral TLE; otherwise, unilateral TLE.

\section{Structural MRI}

MRI acquisition was performed using a $1.5 \mathrm{~T}$ clinical whole body MRI scanner (Siemens Essenza, Erlangen, Germany) at Beijing 466 Hospital, with a standard head coil. T1-weighted MRI images were acquired using FLASH 2D sequence (19 axial slices, repetition time $[\mathrm{TR}]=201 \mathrm{~ms}$, echo time $[\mathrm{TE}]=4.76 \mathrm{~ms}$, flip angle $=70^{\circ}$, matrix $=256 \times 256$, field of view $[\mathrm{FOV}]=194 \times 230$, slice thickness $=5 \mathrm{~mm}$, and scan time $=91$ seconds). T2-weighted MRI images were acquired using Spin Echo sequence (19 axial slices, $\mathrm{TR}=4500 \mathrm{~ms}, \mathrm{TE}=107 \mathrm{~ms}$, flip angle $=70^{\circ}$, 
matrix $=256 \times 256$, FOV $=201 \times 230$, slice thickness $=5 \mathrm{~mm}$, and scan time $=87$ seconds).

MRI images were analyzed by neuroradiologists at Beijing 466 Hospital. The criteria for a diagnosis of HS included the presence of unilateral atrophy and high T2 signal intensity in the hippocampus. ${ }^{5}$ A patient with lesional MRI findings was considered MRI-positive; a patient with non-lesional MRI findings, MRI-negative.

\section{Single voxel 'H-MRS}

Single voxel ${ }^{1} \mathrm{H}-\mathrm{MRS}$ was carried out by the same $1.5 \mathrm{~T}$ Siemens Magnetom Essenza MR scanner with a standard head coil. Transversal and coronal T2-weighted images in three orthogonal planes were used as localizer. The voxel comprised the major part of the hippocampus and covered the visible lesion of the hippocampus (if it existed). The voxel size was $3.375 \mathrm{~cm}^{3}$ $(1.5 \mathrm{~cm} \times 1.5 \mathrm{~cm} \times 1.5 \mathrm{~cm})$. Water suppression was achieved by using chemical shift selective (CHESS) pulses before the "point resolved spectroscopy" (PRESS) localization: TR=4500 ms; $\mathrm{TE}=107 \mathrm{~ms}$; averages $=192$; vector size $=1024$; scan time $=16$ minutes 30 seconds.

The ${ }^{1} \mathrm{H}-\mathrm{MRS}$ data was analyzed with the software provided by Siemens on the Siemens MRI scanner. Resonance intensities were computed by integrating the peak areas of three metabolites: N-acetyl aspartate (NAA), creatine $(\mathrm{Cr})$, and choline (Cho). Ratios of NAA/(Cho $+\mathrm{Cr}$ ) and NAA/Cr were calculated for each patient.

To measure the degree of asymmetry, the asymmetry index (AI) was computed for ${ }^{1} \mathrm{H}-\mathrm{MRS}$ as follows:

$\mathrm{AI}=($ right - left $) /[($ right + left $) / 2] \times 100 \%$,

where (right - left) means the difference between the voxels in the right and left hippocampus. An AI threshold of $12 \%$ was applied to identify significant NAA reduction and Cho or $\mathrm{Cr}$ increase ipsilateral to the seizure focus ${ }^{16} \mathrm{Hemispheric}$ lateralization was based on significant asymmetry of NAA reduction in any of the three NAA measures, ie, AI of $\{[\mathrm{NAA}],[\mathrm{NAA} / \mathrm{Cr}]$, or $[\mathrm{NAA} /(\mathrm{Cho}+\mathrm{Cr})]\} \geq 12 \%$.

\section{IcEEG}

When the findings of noninvasive tests were disconcordant and non-localizing (without clear seizure focus/onset/origin), presurgical icEEG was performed. In total, icEEG monitoring was performed for 12 patients presurgery to lateralize and localize the seizure focus. According to each patient's condition, up to four subdural $2 \times 8$ contact strips were placed in the bilateral temporal and/or frontal (or parietal) regions.
In addition, intraoperative icEEG monitoring was performed at the beginning of and throughout epilepsy surgery for every patient (who underwent surgery) to further localize and confirm the epileptogenic zone. If intraoperative icEEG monitoring confirmed the epileptogenic zone identified by other modalities (presurgical MRI, MRS, EEG, or icEEG) or detected new seizure focus nearby, then the resection region was determined by the intraoperative icEEG findings, and resection was performed accordingly to remove the seizure focus as completely as possible.

\section{Histopathological examination}

Specimens obtained from epilepsy surgery were sent to the Department of Pathology, Beijing Haidian Hospital for histopathological examination after the surgery. The histopathological findings were used to compare with the neuroimaging results and to understand the histopathological alterations and the causes of abnormalities that MRI, ${ }^{1} \mathrm{H}-\mathrm{MRS}$, and EEG indicated (or failed to indicate).

\section{Statistical analysis}

Presurgical findings of different modalities were compared with the seizure focus (detected by icEEG) or the surgical site, and scores of correct lateralization were assigned to each patient for each modality. Spearman correlation between the presurgical findings (eg, the lateralization scores of EEG, MRI, MRS including AIs of MRS metabolites, and icEEG), and the surgical outcome was computed by SPSS 21.0 (IBM Corporation, Armonk, NY, USA).

\section{Results}

Of the 24 patients, 16 had seizure focus in the temporal lobe alone, the other eight patients had seizure foci in the temporal lobe as well as extratemporal regions (Table 1). Based on EEG and/or icEEG findings, 12 patients were classified as unilateral and 12 as bilateral TLE cases. Of the patients with unilateral TLE, seven were MRI-positive with reported temporal lobe abnormality (eg, HS), and five were MRI-negative. Of the patients with bilateral TLE, four were MRI-positive and eight were MRI-negative. IcEEG was performed in 12 patients, including two unilateral MRI-negative, three bilateral MRI-positive, and seven bilateral MRI-negative TLE patients. A total of 23 patients underwent surgery, and one patient (patient 14) did not (due to possible improvement by further antiepileptic drug treatment).

Figure 1 shows the ${ }^{1} \mathrm{H}-\mathrm{MR}$ spectrum and MRI of individual patients. MRI and ${ }^{1} \mathrm{H}-\mathrm{MRS}$ both indicated concordant lateralization to EEG findings in $100 \%$ (7/7) of 
Table I Patient characteristics and subgrouping

\begin{tabular}{|c|c|c|c|c|c|c|c|c|c|}
\hline Pt no & $\begin{array}{l}\text { Sex/agel } \\
\text { duration } \\
\text { (years) }\end{array}$ & MRI & $\begin{array}{l}\text { MRS } \\
\text { (ipsilat/ } \\
\text { contra) }\end{array}$ & $\begin{array}{l}\text { Other } \\
\text { imaging }\end{array}$ & $\begin{array}{l}\text { EEG } \\
\text { (IED/ictal) }\end{array}$ & $\begin{array}{l}\text { icEEG } \\
\text { (IED/ictal) }\end{array}$ & $\begin{array}{l}\text { Seizure } \\
\text { focus } \\
\text { (resection) }\end{array}$ & $\begin{array}{l}\text { Histopathological } \\
\text { findings }\end{array}$ & $\begin{array}{l}\text { Outcome } \\
\text { (Engel) }\end{array}$ \\
\hline \multicolumn{10}{|c|}{ MRI-positive unilateral TLE } \\
\hline 1 & $M / 22 / 10$ & R HS & $\mathrm{NAA} \downarrow \mathrm{Cr} \uparrow$ & & $\mathrm{R}$ temp/R temp & & $\begin{array}{l}R \text { temp and } \\
\text { front }\end{array}$ & $\mathrm{RHS}+\mathrm{MCD}$ & 1 \\
\hline 2 & $M / 22 / 16$ & L HS & $\mathrm{NAA} \downarrow$ & & $L$ temp/L temp & & $\mathrm{L}$ temp & L HS + MCD & I \\
\hline 3 & $F / 28 / 15$ & R HS & $\mathrm{NAA} \downarrow \mathrm{Cr} \uparrow$ & & $\begin{array}{l}\mathrm{R} \text { temp and } \\
\text { front } / \mathrm{R} \text { temp }\end{array}$ & & $\begin{array}{l}\mathrm{R} \text { temp and } \\
\text { front }\end{array}$ & $\mathrm{R} \mathrm{HS}+\mathrm{MCD}$ & 1 \\
\hline 4 & $M / 13 / 10$ & L TA & $\mathrm{NAA} \downarrow \mathrm{Cr} \uparrow$ & & $\begin{array}{l}B \text { temp and } \\
L \text { front } / L \text { temp }\end{array}$ & & $\mathrm{L}$ temp & $\mathrm{L}$ tumor & I \\
\hline 5 & $M / 54 / 26$ & L HA & $\mathrm{NAA} \downarrow \mathrm{Cr} \uparrow$ & & $\mathrm{L}$ temp/L temp & & $L$ temp & L MCD & I \\
\hline 6 & $\mathrm{~F} / 40 / \mathrm{I3}$ & L HA & $\begin{array}{l}\text { NAA } \downarrow / \\
\text { Cho } \uparrow\end{array}$ & & $\begin{array}{l}\mathrm{B} \text { temp and } \\
\mathrm{R} \text { front } / \mathrm{L} \text { temp } \\
\text { and front }\end{array}$ & & $L$ temp & L HS + MCD & 1 \\
\hline 7 & $M / 38 / 28$ & R HA & $\mathrm{NAA} \downarrow \mathrm{Cr} \uparrow$ & & B temp/R temp & & $\mathrm{R}$ temp & $\mathrm{R} H S+M C D$ & II \\
\hline \multicolumn{10}{|c|}{ MRI-positive bilateral TLE } \\
\hline 8 & $M / 14 / 9$ & B HS & /NAA $\downarrow$ & & $\begin{array}{l}B \text { temp } / B \text { temp } \\
\text { ( } L \text { origin) }\end{array}$ & & $L$ temp & L HS + MCD & II \\
\hline 9 & $M / 19 / 15$ & L HA & $\begin{array}{l}\text { NAA } \downarrow C h o \uparrow / / \\
\text { NAA } \downarrow\end{array}$ & & $\mathrm{L}$ temp/B temp & $\begin{array}{l}\mathrm{L} \text { temp/B } \\
\text { temp }(\mathrm{L})\end{array}$ & $L$ temp & $\mathrm{L} \mathrm{HS}+\mathrm{MCD}$ & 1 \\
\hline 10 & $M / 23 / 9$ & L HA & $\begin{array}{l}\mathrm{NAA} \downarrow \mathrm{Cr} \uparrow / \\
\mathrm{NAA} \downarrow\end{array}$ & & $\begin{array}{l}\text { B temp and } \\
\text { front/B front }\end{array}$ & $\begin{array}{l}B \text { temp and } \\
\text { front/L temp } \\
\text { and front }\end{array}$ & $\begin{array}{l}L \text { temp and } \\
\text { front }\end{array}$ & L HS + MCD & II \\
\hline 11 & $M / 28 / 28$ & B TA & $\mathrm{NAA} \downarrow$ & & $\begin{array}{l}\text { B temp and } \\
\text { front/B temp } \\
\text { (L origin) }\end{array}$ & $\begin{array}{l}\text { B temp/ } \\
\text { B temp }(\mathrm{L})\end{array}$ & $\begin{array}{l}\text { B temp } \\
\text { (L temp) }\end{array}$ & L MCD & II \\
\hline \multicolumn{10}{|c|}{ MRI-negative unilateral TLE } \\
\hline 12 & $\mathrm{M} / 9 / 7$ & 0 & $\begin{array}{l}\text { Cho } \uparrow / \\
\text { NAA } \downarrow\end{array}$ & & $\begin{array}{l}B \text { temp } / L \text { temp } \\
\text { and front }\end{array}$ & & $\begin{array}{l}L \text { temp and } \\
\text { front }\end{array}$ & L HS + MCD & 1 \\
\hline 13 & $\mathrm{~F} / 3 \mathrm{I} / 20$ & 0 & $\mathrm{NAA} \downarrow$ & & $\begin{array}{l}\text { B temp and } \\
\text { front/L temp } \\
\text { and front }\end{array}$ & $\begin{array}{l}\text { B temp/ } \\
\text { L temp }\end{array}$ & $L$ temp & $\mathrm{LHS}+\mathrm{MCD}$ & I \\
\hline 14 & $M / 19 / 6$ & 0 & $\mathrm{NAA} \downarrow \mathrm{Cr} \uparrow$ & & $\mathrm{L}$ temp/L temp & & $\mathrm{L}$ temp $(\mathrm{Na})$ & $\mathrm{Na}$ & $\mathrm{Na}$ \\
\hline 15 & $\mathrm{~F} / \mathrm{I} / \mathrm{2}$ & 0 & $\mathrm{NAA} \downarrow \mathrm{Cr} \uparrow$ & & $\begin{array}{l}B \text { temp and } \\
R \text { parietal/L temp }\end{array}$ & & $L$ temp & L HS + MCD & 1 \\
\hline 16 & $M / 28 / 5$ & 0 & $\begin{array}{l}\text { Cho } \uparrow / \\
\text { NAA } \downarrow\end{array}$ & & $\begin{array}{l}B \text { temp and } \\
\text { front } / L \text { temp }\end{array}$ & $\begin{array}{l}\mathrm{L} \text { front } / \mathrm{L} \text { front } \\
\text { and temp }\end{array}$ & $\begin{array}{l}\mathrm{L} \text { front and } \\
\text { temp ( } \mathrm{L} \text { front })\end{array}$ & L MCD & I \\
\hline \multicolumn{10}{|c|}{ MRI-negative bilateral TLE } \\
\hline 17 & $M / 33 / 22$ & 0 & $\begin{array}{l}\text { Cho } \uparrow / / \\
\text { NAA } \downarrow\end{array}$ & & B temp/B temp & $\begin{array}{l}\text { B temp/ } \\
\text { R temp }\end{array}$ & $\begin{array}{l}\text { B temp } \\
\text { (R temp) }\end{array}$ & $\mathrm{RHS}+\mathrm{MCD}$ & III \\
\hline 18 & $\mathrm{~F} / 42 / 10$ & 0 & $\begin{array}{l}\text { Cho } \uparrow / \\
\text { NAA } \downarrow\end{array}$ & & $L$ temp/B temp & & $\begin{array}{l}\mathrm{L} \text { temp and } \\
\text { front }\end{array}$ & L HS & I \\
\hline 19 & $M / 29 / 3$ & 0 & $\mathrm{NAA} \downarrow \mathrm{Cr} \uparrow$ & & $\begin{array}{l}R \text { temp and front/ } \\
R \text { temp }\end{array}$ & $\begin{array}{l}\text { B temp/ } \\
\text { B temp }(\mathrm{L})\end{array}$ & $\begin{array}{l}\text { B temp } \\
(\mathrm{L} \text { temp) }\end{array}$ & L MCD & I \\
\hline 20 & $F / 24 / 22$ & 0 & /NAA $\downarrow$ & $\begin{array}{l}\text { PET: B } \\
\text { temp } \downarrow(R \downarrow)\end{array}$ & $\begin{array}{l}\text { B temp and front/ } \\
\text { B temp and front }\end{array}$ & $\begin{array}{l}\text { B temp/ } \\
\text { R temp }\end{array}$ & $\mathrm{R}$ temp & $\mathrm{R} \mathrm{HS}+\mathrm{MCD}$ & II \\
\hline 21 & $M / 30 / 22$ & 0 & $\begin{array}{l}\text { NAA } \downarrow C h o \uparrow / \\
\text { NAA } \downarrow\end{array}$ & & $\begin{array}{l}B \text { temp and } \\
L \text { front } / B \text { temp }\end{array}$ & $\begin{array}{l}\text { B temp/ } \\
\text { B temp }\end{array}$ & $\begin{array}{l}\text { B temp } \\
\text { (L temp) }\end{array}$ & L MCD & I \\
\hline 22 & $F / 28 / 15$ & 0 & $\begin{array}{l}\text { NAA } \downarrow / \\
\text { NAA } \downarrow\end{array}$ & $\begin{array}{l}\text { SPECT (ictal): } \\
\text { L temp }\end{array}$ & $\begin{array}{l}B \text { temp and } \\
R \text { front } / L \text { temp }\end{array}$ & $\begin{array}{l}\text { B temp/ } \\
\text { R temp }\end{array}$ & $\begin{array}{l}\mathrm{R} \text { temp and } \\
\text { front }\end{array}$ & $\mathrm{RHS}+\mathrm{MCD}$ & IV \\
\hline 23 & $M / 13 / 8$ & 0 & NAA $\downarrow$ Cho $\uparrow$ & & $\begin{array}{l}\text { B temp and front/ } \\
\text { B temp }\end{array}$ & $\begin{array}{l}\text { B temp/ } \\
\text { L temp }\end{array}$ & $\mathrm{L}$ temp & L HS + MCD & II \\
\hline 24 & $F / 32 / 25$ & 0 & Cho $\uparrow / N A A \downarrow$ & & $\begin{array}{l}\text { B temp and front/ } \\
\text { B temp (R) }\end{array}$ & $\begin{array}{l}\mathrm{B} \text { temp }(\mathrm{R}) / \\
\mathrm{B} \text { temp and } \\
\text { parietal }(\mathrm{R})\end{array}$ & $\begin{array}{l}\mathrm{R} \text { temp and } \\
\text { parietal }\end{array}$ & R calcification & 1 \\
\hline
\end{tabular}

Notes: The metabolites with $\mathrm{Al} \geq 0.12$ are reported. NAA $\downarrow$ represents the $\mathrm{Al}$ of (NAA/[Cho $+\mathrm{Cr}])$ or $(\mathrm{NAA} / \mathrm{Cr})$ or $(\mathrm{NAA}) \geq 0.12$.

Abbreviations: Pt, patient; MRI, magnetic resonance imaging; MRS, magnetic resonance spectroscopy; ipsilat, ipsilateral; contra, contralateral; EEG, electroencephalogram; PET, positron emission tomography; SPECT, single-photon emission computed tomography; IED, interictal epileptiform discharge; ictal, ictal activity; Engel, Engel classification; icEEG, intracranial EEG; TLE, temporal-lobe epilepsy; M, male; F, female; L, left; R, right; B, bilateral; HS, hippocampal sclerosis; TA, temporal abnormality; HA, hippocampal abnormality; 0, negative-MRI; NAA, N-acetyl aspartate; Cho, choline; Cr, creatine; temp, temporal lobe; front, frontal lobe; parietal, parietal lobe; MCD, malformation of cortical development; $\mathrm{Na}$, not available due to non-surgery performed (for one patient); Al, asymmetry index. 
patients with unilateral MRI-positive TLE, and they indicated concordant lateralization to icEEG findings in $75 \%(3 / 4)$ of patients with bilateral MRI-positive TLE. In addition, ${ }^{1} \mathrm{H}-\mathrm{MRS}$ indicated concordant lateralization to EEG and/or icEEG findings in $60 \%(3 / 5) \sim 75 \%$ (3/4) of patients with unilateral MRI-negative TLE, and concordant lateralization to icEEG findings in $50 \%(4 / 8)$ of patients with bilateral MRI-negative TLE. The $60 \%-75 \%$ concordance between ${ }^{1} \mathrm{H}-\mathrm{MRS}$ and EEG/icEEG in unilateral MRI-negative TLE was due to one patient (case 16) who was also diagnosed with ETLE (EEG and icEEG found abnormal epileptiform discharges in both the left frontal and temporal regions, with more abnormal electrical discharges in the left frontal region). If case 16 was counted as unilateral MRI-negative TLE, the ${ }^{1} \mathrm{H}-\mathrm{MRS}$ and EEG/icEEG concordance was $60 \%$; otherwise, $75 \%$.

In addition, patients with unilateral TLE achieved good outcome: 90.9\% (11/12) were seizure-free (Engel class I)
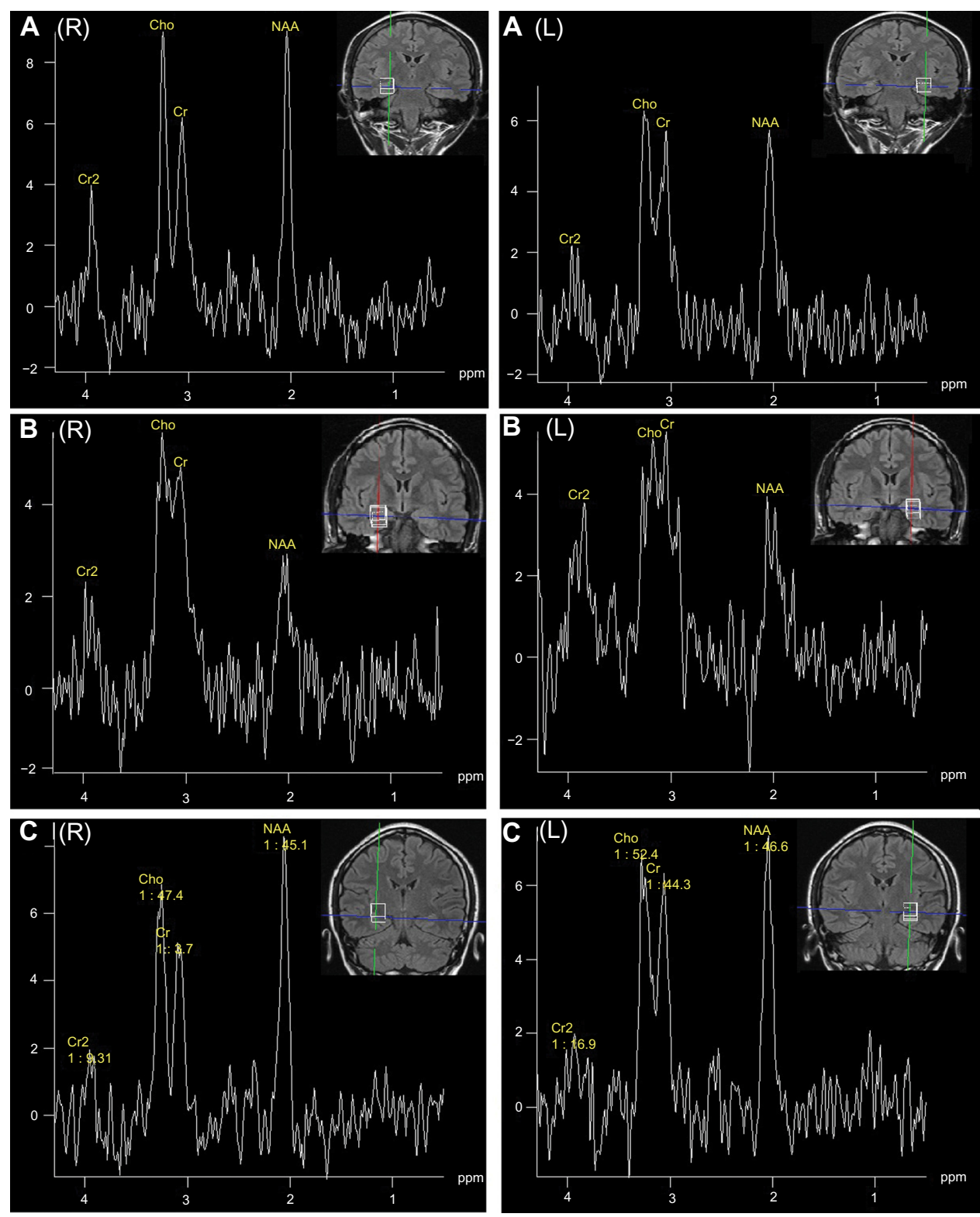

Figure I 'H-MR spectrum and MRI of individual patients. (A) 'H-MR spectrum and MRI of patient I 3 (3I-year-old female; seizure history, 20 years; right [NAA/Cho + Cr] =0.48, left $[\mathrm{NAA} / \mathrm{Cho}+\mathrm{Cr}]=0.42$; seizure focus in the left temporal region; left HS; surgical outcome, Engel class I). (B) 'H-MR spectrum and MRI of patient 7 (38 year old male; seizure history, 28 years; right $[\mathrm{NAA} /(\mathrm{Cho}+\mathrm{Cr})]=0.26$, left $[\mathrm{NAA} /(\mathrm{Cho}+\mathrm{Cr})]=0.35$; seizure focus in the right temporal region; right $\mathrm{HS}$; surgical outcome, Engel class II). (C) 'H-MR spectrum and MRI of patient 20 (24-year-old female; seizure history, 22 years; right [NAA/Cho $+\mathrm{Cr}]=0.56$, left [NAA/Cho $+\mathrm{Cr}]=0.48$; seizure focus in the bilateral temporal regions; right HS; surgical outcome, Engel class II).

Abbreviations: R, right; L, left; 'H-MR, proton magnetic resonance; MRI, magnetic resonance imaging; NAA, N-acetyl aspartate; Cho, choline; Cr, creatine; HS, hippocampal sclerosis; ppm, parts per million. 
(among them, 85.7\% [6/7] with MRI-positive and 100\% [5/5] with MRI-negative were seizure-free), and the rest of the patients had Engel class II outcome. However, in patients with bilateral TLE, 41.7\% (5/12) became seizure-free (including one [25\%] MRI-positive and four [50\%] MRI-negative patients); and all (4/4, 100\%) MRI-positive and six (6/8, $75 \%$ ) MRI-negative patients had Engel class I or II outcome. Although $25 \%(2 / 8)$ of patients with MRI-negative bilateral TLE attained unfavorable outcomes (Engel class III or IV), $50 \%$ (4/8) of them did become seizure-free (Figure 2).

Correlation results are summarized in Table 2. For all patients who underwent surgery $(n=23)$, EEG lateralization score was significantly $(P<0.05)$ correlated with the seizure outcome, and for those who underwent presurgical icEEG ( $n=12)$, icEEG lateralization score was significantly correlated with the outcome. For patients with positive MRI $(n=11)$, only MRI lateralization score was significantly correlated with the seizure outcome, while for those with negative-MRI $(n=12)$, EEG lateralization finding (potentially together with icEEG finding, $P<0.1$ ) was significantly $(P<0.05)$ correlated with the outcome.

Further, compared with non-seizure-free patients, the seizure-free patients had a lower percentage of contralateral NAA reduction $(40 \%$ [6/15] versus $62.5 \%$ [5/8], the difference was not significant), and the individual asymmetry levels of individual ${ }^{1} \mathrm{H}$-MRS measures were not correlated with seizure outcome. In addition, the ipsilateral NAA/(Cho $+\mathrm{Cr}$ ) values in non-seizure-free or seizure-free patients were not significantly lower than those of contralateral values, and the ipsilateral NAA/ $(\mathrm{Cho}+\mathrm{Cr})$ values of non-seizure-free patients were not lower than those of seizure-free patients.

\section{Discussion}

Focus lateralization in TLE is still challenging especially in non-lesional and bilateral TLE. This study showed that: 1) ${ }^{1} \mathrm{H}-\mathrm{MRS}$ had high concordant lateralization to video-EEG and/or icEEG findings in MRI-positive unilateral TLE (100\%), moderate concordance in MRI-positive bilateral TLE (75\%) and MRI-negative unilateral TLE (60\%-75\%), and low concordance in MRI-negative bilateral TLE (50\%); 2) a higher percentage of patients with unilateral TLE than that of those with bilateral TLE (90.9\% versus $41.7 \%)$ became seizure-free, the worst outcome was seen in patients with MRI-negative bilateral TLE; and 3) EEG and icEEG lateralization findings (together with MRI findings in MRIpositive patients) were associated with seizure outcome, and there was a lower percentage of contralateral NAA reduction in the seizure-free patients than in those with non-seizurefree outcome.

\section{Focus lateralization with a multimodal approach in challenging TLE cases}

Up to $30 \%$ of patients with TLE have normal MRI findings. ${ }^{13}$ The results of this study suggest that the ${ }^{1} \mathrm{H}$-MRS has a much lower accuracy of focus lateralization in MRI-negative TLE than in MRI-positive TLE (60\%-75\% versus 100\%). In

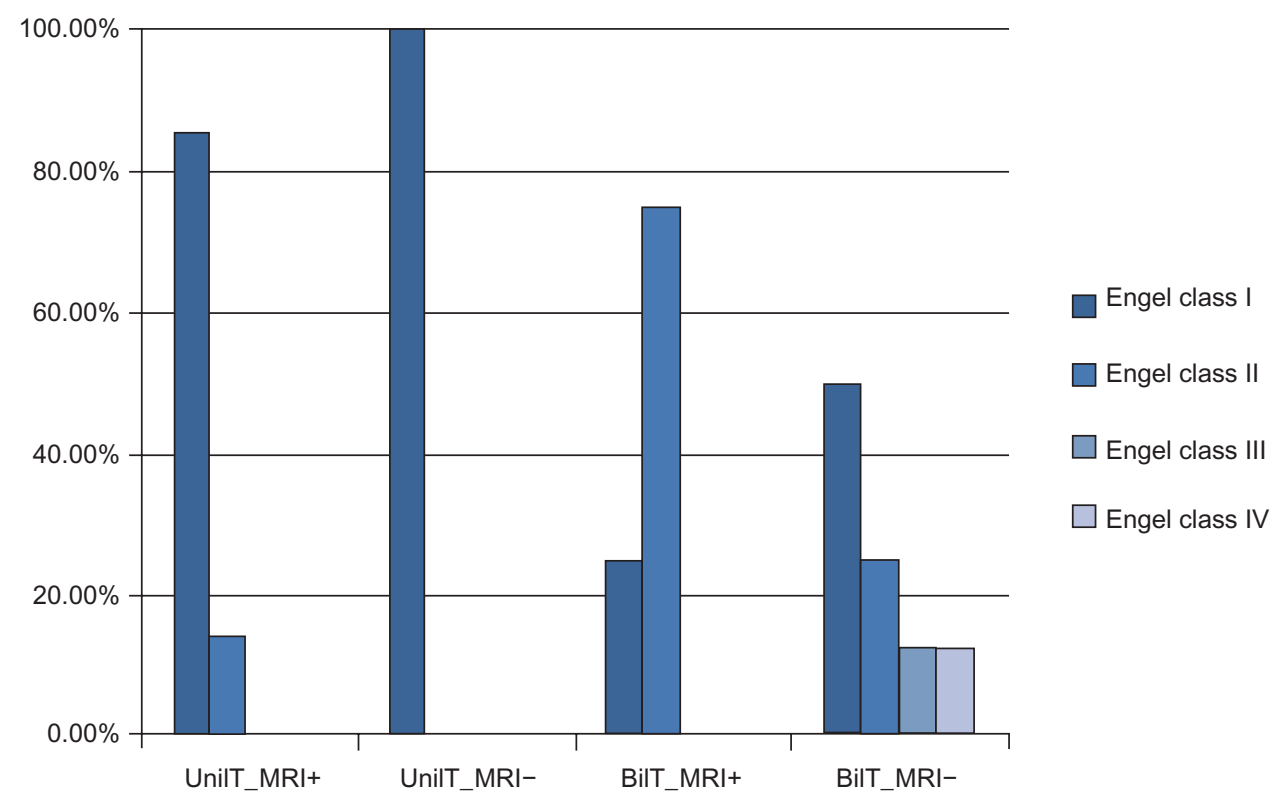

Figure 2 Surgical outcome of unilateral or bilateral MRI-positive or negative TLE $(n=23)$.

Abbreviations: UnilT_MRI+, unilateral TLE with positive MRI ( $n=7)$; UnilT_MRI-, unilateral TLE with negative-MRI ( $n=4)$; BilT_MRI+, bilateral TLE with positive MRI ( $n=4)$; BilT_MRI-, bilateral TLE with negative-MRI $(n=8)$; MRI, magnetic resonance imaging; TLE, temporal-lobe epilepsy. 
Table 2 Spearman correlation between presurgical (and pathological) findings and surgical outcome

\begin{tabular}{llll}
\hline & \multicolumn{3}{l}{ Surgical outcome (Engel classification: I-IV) } \\
\cline { 2 - 4 } & $\begin{array}{l}\text { All patients } \\
(\mathbf{n}=\mathbf{2 3})\end{array}$ & $\begin{array}{l}\text { Patients with } \\
\text { positive MRI } \\
(\mathbf{n}=\mathbf{I} \mathbf{)})\end{array}$ & $\begin{array}{l}\text { Patients with } \\
\text { negative- } \\
\text { MRI }(\mathbf{n}=\mathbf{I})\end{array}$ \\
\hline Age & -0.05 & -0.30 & $-0.1 \mathrm{I}$ \\
Sex & $-0.1 \mathrm{I}$ & -0.36 & 0.03 \\
Duration & 0.23 & 0.00 & 0.31 \\
of seizure & & & \\
EEG_lat & -0.47 & -0.18 & -0.67 \\
MRI_lat & $(P=0.025)^{\mathrm{a}}$ & & $(P=0.018)^{\mathrm{a}}$ \\
MRS_lat & -0.28 & $-0.62(P=0.040)^{\mathrm{a}}$ & -0.21 \\
icEEG_lat $(\mathrm{n}=12)$ & -0.20 & -0.42 & -0.14 \\
& 0.44 & 0.39 & $0.56(P=0.056)^{\mathrm{b}}$ \\
HS or not ${ }^{\mathrm{c}}$ & $(P=0.036)^{\mathrm{a}}$ & & 0.49 \\
\hline Notes:Due & 0.30 & 0.04 &
\end{tabular}

Notes: Due to the fuzziness and difficulty of combining the MRS data (NAA, Cr, Cho, etc), two sets of combination rules were applied. The value of correct MRS lateralization is the highest correlation coefficients (either positive or negative) of the two MRS lat determined by the two MRS combination rules. ${ }^{a}$ Correlation coefficients were at two-tailed significance $(P<0.05)$; ${ }^{b}$ correlation coefficients were at one-tailed significance $(P<0.10)$; ' whether the patient has HS in the surgical site, which is determined by pathological findings.

Abbreviations: MRI, magnetic resonance imaging; EEG, electroencephalogram EEG_lat, correct lateralization of EEG compared with the surgical site; MRI_lat, correct lateralization of MRI; MRS, magnetic resonance spectroscopy; MRS lat, correct lateralization of MRS; icEEG, intracranial EEG; icEEG_lat, correct lateralization of icEEG; HS, hippocampus sclerosis; NAA, N-acetyl aspartate; Cho, choline; $\mathrm{Cr}$, creatine.

MRI-negative unilateral TLE, the moderate concordance (60\%-75\%) of ${ }^{1} \mathrm{H}-\mathrm{MRS}$ to EEG findings (or $25 \%-40 \%$ disconcordance) requires more evidence from neuroimaging or icEEG for hemispheric lateralization. In this study, ictal icEEG findings largely confirmed the ictal EEG findings in MRI-negative unilateral TLE. In addition, other neuroimaging modalities such as PET may be considered to aid focus lateralization in MRI-negative TLE.

Compared with unilateral TLE, focus lateralization in bilateral TLE remains a bigger challenge. Cendes et al reported that ${ }^{1} \mathrm{H}$-MRS correctly lateralized $86 \%$ in 100 consecutive (mixed) TLE cases, but 54\% with bilaterally abnormal TLE. ${ }^{17}$ In patients with negative-MRI, this study indicated that ${ }^{1} \mathrm{H}$-MRS was still helpful for focus lateralization in unilateral TLE $(60 \%-75 \%)$, but not in bilateral TLE $(50 \%)$, which is consistent with the findings of two other studies. ${ }^{5,18}$ Because of the difficulty in identifying the side of seizure origin ${ }^{13}$ and the often unfavorable outcome, ${ }^{12}$ patients with bilateral TLE are often excluded from surgical treatment. ${ }^{11,14}$ Presurgical icEEG is critical for focus lateralization and localization in bilateral TLE. In this study, icEEG monitoring was performed in most (83.3\%) patients with bilateral TLE: among them, 75\% (3/4) were MRI-positive and 87.5\% (7/8) were MRI-negative. Overall, 100\% (4/4) of patients with
MRI-positive bilateral TLE attained good outcomes (Engel class I or II), while patients with MRI-negative bilateral TLE attained varied outcomes: 75\% (6/8) had good outcomes, and $25 \%(2 / 8)$ had bad outcomes (Engel class III or IV).

Since false localization rate by MRI, MRS, or EEG alone may be high, a multimodal imaging approach including MRI, MRS, EEG, and PET/single-photon emission computed tomography (SPECT) is useful to increase the diagnostic yield in challenging TLE cases such as non-lesional or bilateral TLE. ${ }^{5-7}$ Concordant neuroimaging findings could not only guide the placement of icEEG electrodes, but also help avoid the need for invasive icEEG monitoring. For example, due to limited spatial sampling of icEEG, false negative icEEG findings have been reported, ${ }^{6}$ and concordant neuroimaging and EEG findings might guide the surgery and minimize the need for presurgical icEEG in such cases.

Focus lateralization and localization in ETLE is another challenge. Eight $(8 / 24,33.3 \%)$ patients in this study had unilateral seizure foci in both the temporal and extratemporal regions. Three of those $(3 / 8,37.5 \%)$ were MRI-positive and could be correctly lateralized by ${ }^{1} \mathrm{H}-\mathrm{MRS}$. In the rest of those with negative-MRI, only one $(1 / 5,20 \%)$ was correctly lateralized by ${ }^{1} \mathrm{H}-\mathrm{MRS}$. Such accuracy was much lower than that in patients with seizure focus in the temporal region alone. Seizure foci in both the temporal and extratemporal regions might cause widespread neuron loss and a more diffuse distribution of ${ }^{1} \mathrm{H}$-MRS abnormality. Mueller et al studied the extrahippocampal ${ }^{1} \mathrm{H}-\mathrm{MRS}$ abnormalities in TLE, ${ }^{20,21}$ and found that 1) extrahippocampal NAA/ $(\mathrm{Cr}+\mathrm{Cho})$ reductions had a bilateral fronto-temporal distribution in TLE with mesial temporal sclerosis (MTS) and a more diffuse distribution in TLE without MTS; and 2) the inter-individual variability and non-focal and inhomogeneous features of extrahippocampal NAA/(Cr+Cho) reductions reduced their value in seizure focus lateralization. ${ }^{21}$

To lateralize and localize seizure focus in challenging TLE, long-term extensive video-EEG monitoring and icEEG are helpful. Video-EEG could increase the diagnostic yield of focus lateralization and localization, ${ }^{22}$ and is successful in focus localization in $60 \%-90 \%$ of cases. ${ }^{23,24}$ Intensive 24 -hour high-resolution video-EEG monitoring has even been proposed as the "gold standard" for focus localization, ${ }^{23,24}$ and such "gold standard" has been used in a number of studies. ${ }^{5,18}$ Cendes further suggested that when the interictal EEG and hippocampal atrophy coincide, ictal EEG recording might not be mandatory in TLE focus lateralization and localization. ${ }^{17}$ However, the results of this study indicated that video-EEG is good for focus localization, but it might not be sufficient 
as the "gold standard," and icEEG is still needed in difficult cases such as nonlesional or bilateral TLE.

Taken together, a multimodal approach using neuroimaging, EEG and/or icEEG is needed in presurgical assessment, and it provides surgical options for patients with challenging TLE cases.

\section{The sensitivity and predictive value of ' $\mathrm{H}$-MRS}

${ }^{1} \mathrm{H}-\mathrm{MRS}$ has been increasingly used as a noninvasive "biopsy" tool for detection of metabolite hippocampal abnormalities in TLE presurgical evaluation. NAA reduction indicates neuronal loss or dysfunction; $\mathrm{Cr}$ increase indicates increased astrocytes and glial cells; and Cho increase may reflect cell membrane damage, myelin breakdown, and gliosis. ${ }^{13}$

The AI threshold (12\%) used in this study to identify metabolite abnormalities and lateralize the affected hemisphere was the same as that in Knowlton et al, ${ }^{16}$ where $61 \%$ ( ${ }^{1} \mathrm{H}-\mathrm{MRS}$ sensitivity for focus lateralization in MRI-negative unilateral TLE) was obtained, which is comparable to the sensitivity (60\%-75\%) (in MRI-negative unilateral TLE) obtained in this study and other studies (eg, 55\%-65\% by Connelly et al). ${ }^{25}$ If relaxing the lateralization criteria by, eg, lowering the AI threshold, or adding Cr or Cho increase (ipsilateral to the seizure focus) to the lateralization criteria to capture metabolite abnormalities, ${ }^{1} \mathrm{H}-\mathrm{MRS}$ might be more sensitive in hemispheric lateralization, but we agree with Knowlton et al that a conservative ${ }^{1} \mathrm{H}-\mathrm{MRS}$ lateralization criteria is needed, even at the expense of sensitivity. ${ }^{16}$

Since ${ }^{1} \mathrm{H}$-MRS has been widely used for seizure focus lateralization, ${ }^{5,11,17,25,26} \mathrm{MCD}$ abnormality detection, ${ }^{27}$ and brain tumor grading, ${ }^{19,28,29}$ it is natural to expect that ${ }^{1} \mathrm{H}-\mathrm{MRS}$ is sensitive enough to detect $\mathrm{HS}$ and even differentiate between HS, MCD, and tumor. However, the results of this study did not support this. Chernov et al also reported that ${ }^{1} \mathrm{H}-\mathrm{MRS}$ had limited usefulness for differentiation of HS and low-grade brain tumor. ${ }^{11}$ Nevertheless, Vermathen et al demonstrated that hippocampal NAA was able to discriminate neocortical TLE (including MCD) from mesial TLE (including HS). ${ }^{30}$ Hammen et al demonstrated that multi-voxel ${ }^{1} \mathrm{H}-\mathrm{MRS}$ was able to distinguish between mesial TLE and lateral neocortical TLE. ${ }^{31,32}$ In addition, NAA reduction before surgery was found to correlate with the degree of HS, reflecting neuronal loss..$^{33,34}$

The higher percentage of contralateral metabolite abnormalities in non-seizure-free patients (although insignificant) than in seizure-free patients found in this study suggested that ${ }^{1} \mathrm{H}-\mathrm{MRS}$ has a predictive value for seizure outcome. This was supported by a number of studies, with the finding that patients with unilateral metabolite alterations ipsilateral to the seizure focus had better seizure outcome than those with contralateral or bilateral metabolite alterations, ${ }^{11,35}$ suggesting that ${ }^{1} \mathrm{H}-\mathrm{MRS}$ could predict surgical outcome. ${ }^{11,13,24,35,36}$ In addition, this study showed that the MRS findings were not correlated with the outcome, and the ipsilateral NAA/ $(\mathrm{Cho}+\mathrm{Cr})$ values were not significantly lower than those of contralateral values in either non-seizure-free patients or seizure-free patients, suggesting that the predictive power of ${ }^{1} \mathrm{H}-\mathrm{MRS}$ was weak. This was different from the findings of Suhy et al, ${ }^{35}$ and such difference might be due to the different patient samples, different ${ }^{1} \mathrm{H}$-MRS acquisition equipment and parameters, and different surgeries performed for the patients between the two studies. Further, the results of this study indicated that the predictive value of ${ }^{1} \mathrm{H}-\mathrm{MRS}$ was largely reduced in bilateral TLE. This is because one hemisphere in bilateral TLE often initiates most seizures, and the hemisphere with the greatest metabolite alterations may not always be the side of seizure origin. ${ }^{13}$

\section{Predictive value of other presurgical findings for surgical outcome}

The significant correlation between EEG, icEEG, or MRI lateralization findings, and seizure outcome indicate the predictive value of MRI and EEG/icEEG. However, mild lesions such as mild HS and focal cortical dysplasia are difficult to identify on regular MRI. They may be missed by MRI, mis-regarded as non-lesional, and even excluded from presurgical evaluation. Thus, there are controversies on the utility of neuroimaging in predicting surgical outcome. ${ }^{37}$ In this study, the fact that 8 out of 13 MRI-negative patients had HS partially reflected such limitation of MRI.

Nevertheless, the predictive value of neuroimaging for epilepsy surgical outcome has been reported by a number of studies. For example, Lerner et al, ${ }^{38}$ Cossu et al, ${ }^{39}$ WiddessWalsh et $\mathrm{al}^{40}$ and Jeha et $\mathrm{al}^{41}$ have shown that the complete resection of the abnormality detected by preoperative MRI is the most important predictor for favorable postoperative outcome. Functional neuroimaging modalities such as magnetoencephalography (MEG)/magnetic source imaging (MSI), PET, and ictal SPECT also have clinical value in predicting seizure-free outcome. ${ }^{6}$ In addition, systematic reviews and meta-analyses have found that abnormal MRI (lesion such as HS on MRI), EEG abnormalities (well localized EEG), EEG/MRI concordance, and the presence of HS (or MTS) are positive predictors of seizure outcome, while normal MRI, non-localized EEG, and use of icEEG are negative outcome 
predictors. ${ }^{37,42-46}$ These findings support the predictive value of MRI, EEG, and icEEG.

Limitations of single modality such as MRI may lead to limited predictive value for seizure outcome. Therefore, a multimodal approach including multiple neuroimaging modalities such as EEG/ESI (EEG source imaging), MRI, MRS, PET, SPECT, and MEG/MSI is needed not only for focus lateralization and localization in presurgical assessment, but also for improvement of outcome prediction. Further study on this patient data is needed to identify predictors of seizure outcome and gain better understanding of the relationship between presurgical findings and surgical outcome.

\section{Limitations}

There are several limitations in this study. First, PET and SPECT were performed for only $2(8.3 \%)$ patients in presurgical evaluation (Table 1). This was because the local hospital (ie, Beijing Haidian Hospital) does not have a PET or SPECT scanner, and patients who need a PET or SPECT scan have to go to other hospitals (as the MRI and MRS acquisition), which is often time consuming. In such cases, the surgeons tended to use presurgical icEEG to detect the seizure focus instead, which increases the usage of icEEG in presurgical evaluation. Noninvasive neuroimaging such as PET and SPECT could be used more in the future to reduce the need for invasive icEEG and its associated risks and costs. Second, the diagnostic accuracy of MRI was not high in this study: eight out of eleven patients (72.7\%) with positive MRI had HS, one $(0.9 \%)$ had a tumor, and two (18.2\%) had MCD (which were confirmed by histopathological examination), while in MRI-negative TLE, 8 out of 13 patients (61.5\%) had HS. In other words, $38.5 \%$ of the patients with HS were not identified by MRI. The HS that eluded MRI visual inspection might be mild, while the relatively low resolution of the clinical MRI scanner used ( 19 slices, slick thickness $=5 \mathrm{~mm}$ ) and the experiences of the neuroradiologists who examined the MRI might also contribute to the relatively low specificity of MRI in HS identification. A high-resolution MRI scanner and quantitative MRI may be used to better reveal mild HS on MRI in the future. Third, the evaluation of surgical outcome using Engel classification in this study might be subjective and imprecise in several patients who had non-seizure-free outcomes, which might slightly influence the results associated with such outcomes in this study. This may be improved by more detailed patient follow-up over a longer period of time, which could provide more information for postsurgical assessment and make it more objective and accurate in future studies. Finally, the patients in this study were heterogeneous, and the sample sizes in the four subgroups were relatively small $(n=4-8)$. This could be improved by recruiting more patients in each subgroup in the future.

\section{Conclusion}

In summary, this study investigated focus lateralization in TLE and found that ${ }^{1} \mathrm{H}-\mathrm{MRS}$ had relatively high sensitivity in MRI-positive unilateral TLE, moderate sensitivity in MRIpositive bilateral TLE and MRI-negative unilateral TLE, and relatively low sensitivity in MRI-negative bilateral TLE. In addition, $90.9 \%$ of patients with unilateral TLE and $41.7 \%$ of patients with bilateral TLE (50\% MRI-negative bilateral TLE) attained seizure-free outcome. The MRS findings were not correlated with seizure outcome, while non-seizure-free patients had an insignificantly higher percentage of contralateral NAA reduction compared with seizure-free patients, indicating that ${ }^{1} \mathrm{H}-\mathrm{MRS}$ has a relatively low predictive value for surgical outcome. Further, EEG and icEEG findings were correlated with seizure outcome, and for patients with positive MRI, MRI findings were also correlated with seizure outcome, indicating the predictive value of EEG, icEEG, and MRI. Taken together, a multimodality approach including neuroimaging, EEG, and/or icEEG in surgical planning could lateralize and localize seizure focus successfully in most TLE cases, and provide surgical options for patients with non-lesional or bilateral TLE.

\section{Acknowledgments}

We are grateful to Zhao Jianxiu and Du Nansen from the Department of Radiology, Beijing 466 Hospital for their cooperation in acquiring the MRI and MRS data for the patients. We also thank Tan Bojing in the Department of Functional Neurology and Neurosurgery, Beijing Haidian Hospital for his help in providing patient information. This study was partially supported by National Natural Science Foundation (NSF) of the People's Republic of China Grant 81071211.

\section{Disclosure}

The authors report no conflicts of interest in this work.

\section{References}

1. Babb TL. Bilateral pathological damage in temporal lobe epilepsy. Can J Neurol Sci. 1991;18:645-648.

2. Wolf HK, Zentner J, Hufnagel A, et al. Surgical pathology of chronic epileptic seizure disorders: experience with 63 specimens from extratemporal corticectomies, lobectomies and functional hemispherectomies. Acta Neuropathol. 1993;86:466-472.

3. Widjaja E, Raybaud C. Advances in neuroimaging in patients with epilepsy. Neurosurg Focus. 2008;25:E3. 
4. Téllez-Zenteno JF, Dhar R, Wiebe S. Long-term seizure outcomes following epilepsy surgery: a systematic review and meta-analysis. Brain. 2005;128:1188-1198.

5. Doelken MT, Richter G, Stefan H, et al. Multimodal coregistration in patients with temporal lobe epilepsy - results of different imaging modalities in lateralization of the affected hemisphere in MR imaging positive and negative subgroups. AJNR Am J Neuroradiol. 2007;28: 449-454.

6. Knowlton RC, Elgavish RA, Limdi N, et al. Functional imaging: I. Relative predictive value of intracranial electroencephalograpy. Ann Neurol. 2008;64:25-34.

7. Knowlton RC, Elgavish RA, Bartolucci A, et al. Functional imaging: II. Prediction of epilepsy surgery outcome. Ann Neurol. 2008;64:35-41.

8. Shibasaki H, Ikeda A, Nagamine T. Use of magnetoencephalography in the presurgical evaluation of epilepsy patients. Clin Neurophysiol. 2007;118:1438-1448.

9. Blount JP, Cormier J, Kim H, Kankirawatana P, Riley KO, Knowlton RC. Advances in intracranial monitoring. Neurosurg Focus. 2008;25:E18.

10. Duncan JS. Imaging the surgical treatment of epilepsy. Nat Rev Neurol. 2010;6:537-550.

11. Chernov MF, Ochiai T, Ono Y, et al. Role of proton magnetic resonance spectroscopy in preoperative evaluation of patients with mesial temporal lobe epilepsy. J Neurol Sci. 2009;285:212-219.

12. Harroud A, Bouthillier A, Weil AG, Nguyen DK. Temporal lobe epilepsy surgery failures: a review. Epilepsy Res Treat. 2012;2012:201651.

13. Hammen T, Kuzniecky R. Magnetic resonance spectroscopy in epilepsy. In: Stefan H, Theodore WH, editors. Handbook of Clinical Neurology. Vol 107 (3rd series). Epilepsy, Part I. 2012:399-408.

14. Hori T, Yamane F, Ochiai T, et al. Selective subtemporal amygdalohippocampectomy for refractory temporal lobe epilepsy: operative and neuropsychological outcomes. J Neurosurg. 2007;106:134-141.

15. Engel J Jr, Van Ness PC, Rasmussen T, et al. Outcome with respect to epileptic seizures. In: Engel J Jr, editor. Surgical Treatment of the Epilepsies. 2nd ed. New York: Raven Press; 1993:609-622.

16. Knowlton RC, Laxer KD, Ende G, et al. Presurgical multimodality neuroimaging in electroencephalographic lateralized temporal lobe epilepsy. Ann Neurol. 1997;42:829-837.

17. Cendes F, Caramanos Z, Andermann F, et al. Proton magnetic resonance spectroscopic imaging and magnetic resonance imaging volumetry in the lateralization of temporal lobe epilepsy: a series of 100 patients. Ann Neurol. 1997;42:737-746.

18. Hammen T, Kerling F, Schwarz M, et al. Identifying the affected hemisphere by (1)H-MR spectroscopy in patients with temporal lobe epilepsy and no pathological findings in high resolution MRI. Eur $J$ Neurol. 2006;13:482-490.

19. Likavcanova K, Dobrota D, Liptaj T, et al. In vitro study of astrocytic tumour metabolism by proton magnetic resonance spectroscopy. Gen Physiol Biophys. 2005;24:327-335.

20. Mueller SG, Laxer KD, Cashdollar N, Flenniken DL, Matson GB, Weiner MW. Identification of abnormal neuronal metabolism outside the seizure focus in temporal lobe epilepsy. Epilepsia. 2004;45:355-366.

21. Mueller SG, Ebel A, Barakos J, et al. Widespread extrahippocampal $\mathrm{NAA} /(\mathrm{Cr}+\mathrm{Cho})$ abnormalities in TLE with and without mesial temporal sclerosis. J Neurol. 2011;258:603-612.

22. Smith SJM. EEG in the diagnosis, classification, and management of patients with epilepsy. $J$ Neurol Neurosurg Psychiatry. 2005;76: ii2-ii7.

23. Cendes F, Li LM, Watson C, et al. Is ictal recording mandatory in temporal lobe epilepsy? Not when the interictal electroencephalogram and hippocampal atrophy coincide. Arch Neurol. 2000;57:497-500.

24. Stefan H, Pauli E, Eberhardt KE, et al. MRI spectroscopy, T2 relaxometry, and postoperative prognosis in cryptogenic temporal lobe epilepsy. Nervenarzt. 2000;71:282-287.

25. Connelly A, Van Paesschen W, Porter DA, Johnson CL, Duncan JS, Gadian DG. Proton magnetic resonance spectroscopy in MRI-negative temporal lobe epilepsy. Neurology. 1998;51:61-66.
26. Chang KH, Kim HD, Park SW, et al. Usefulness of single voxel proton MR spectroscopy in the evaluation of hippocampal sclerosis. Korean J Radiol. 2000;1:25-32.

27. Woermann FG, McLean MA, Bartlett PA, Barker GJ, Duncan JS. Quantitative short echo time proton magnetic resonance spectroscopic imaging study of malformations of cortical development causing epilepsy. Brain. 2001;124:427-436.

28. Stadlbauer A, Gruber S, Nimsky C, et al. Preoperative grading of gliomas by using metabolite quantification with highspatial-resolution proton MR spectroscopic imaging. Radiology. 2006;238:958-969.

29. Fountas KN, Kapsalaki EZ, Vogel RL, Fezoulidis I, Robinson JS, Gotsis ED. Noninvasive histologic grading of solid astrocytomas using proton magnetic resonance spectroscopy. Stereotact Funct Neurosurg. 2004;82:90-97.

30. Vermathen P, Ende G, Laxer KD, et al. Hippocampal N-acetylaspartate in neocortical epilepsy and mesial temporal lobe epilepsy. Ann Neurol. 1997;42:194-199.

31. Hammen T, Stefan H, Pauli E, et al. 1H-MR spectroscopy: a promising method in distinguishing subgroups in temporal lobe epilepsy? J Neurol Sci. 2003;215:21-25.

32. Riederer F, Bittsansky M, Schmidt C, et al. 1H magnetic resonance spectroscopy at $3 \mathrm{~T}$ in cryptogenic and mesial temporal lobe epilepsy. NMR Biomed. 2006;19:544-553.

33. Duc CO, Trabesinger AH, Weber OM, et al. Quantitative $1 \mathrm{H}$ MRS in the evaluation of mesial temporal lobe epilepsy in vivo. Magn Reson Imaging. 1998;16:969-979.

34. Hammen T, Hildebrandt M, Stadlbauer A, et al. Noninvasive detection of hippocampal sclerosis: correlation between metabolite alterations detected by (1)H-MRS and neuropathology. NMR Biomed. 2008;21:545-552.

35. Suhy J, Laxer KD, Capizzano AA, et al. 1H MRSI predicts surgical outcome in MRI-negative temporal lobe epilepsy. Neurology. 2002;58: 821-823.

36. Kuzniecky R, Hugg J, Hetherington H, et al. Predictive value of 1 H MRSI for outcome in temporal lobectomy. Neurology. 1999;53:694-698.

37. Rowland NC, Englot DJ, Cage TA, Sughrue ME, Barbaro NM, Chang EF. A meta-analysis of predictors of seizure freedom in the surgical management of focal cortical dysplasia. J Neurosurg. 2012;116(5): 1035-1041.

38. Lerner JT, Salamon N, Hauptman JS, et al. Assessment and surgical outcomes for mild type I and severe type II cortical dysplasia: a critical review and the UCLA experience. Epilepsia. 2009;50:1310-1335.

39. Cossu M, Lo Russo G, Francione S, et al. Epilepsy surgery in children: results and predictors of outcome on seizures. Epilepsia. 2008;49: $65-72$.

40. Widdess-Walsh P, Jeha L, Nair D, Kotagal P, Bingaman W, Najm I. Subdural electrode analysis in focal cortical dysplasia: predictors of surgical outcome. Neurology. 2007;69:660-667.

41. Jeha LE, Najm I, Bingaman W, Dinner D, Widdess-Walsh P, Lüders H. Surgical outcome and prognostic factors of frontal lobe epilepsy surgery. Brain. 2007;130:574-584.

42. McIntosh AM, Wilson SJ, Berkovic SF. Seizure outcome after temporal lobectomy: current research practice and findings. Epilepsia. 2001;42(10):1288-1307.

43. Najm I, Jehi L, Palmini A, Gonzalez-Martinez J, Paglioli E, Bingaman W. Temporal patterns and mechanisms of epilepsy surgery failure. Epilepsia. 2013;54(5):772-782.

44. Englot DJ, Rolston JD, Wang DD, Sun PP, Chang EF, Auguste KI. Seizure outcomes after temporal lobectomy in pediatric patients. J Neurosurg Pediatr. 2013;12(2):134-141.

45. Téllez-Zenteno JF, Hernández Ronquillo L, Moien-Afshari F, Wiebe S. Surgical outcomes in lesional and non-lesional epilepsy: a systematic review and meta-analysis. Epilepsy Res. 2010;89(2-3): 310-318.

46. Tonini C, Beghi E, Berg AT, et al. Predictors of epilepsy surgery outcome: a meta-analysis. Epilepsy Res. 2004;62(1):75-87. 


\section{Publish your work in this journal}

Neuropsychiatric Disease and Treatment is an international, peerreviewed journal of clinical therapeutics and pharmacology focusing on concise rapid reporting of clinical or pre-clinical studies on a range of neuropsychiatric and neurological disorders. This journa is indexed on PubMed Central, the 'PsycINFO' database and CAS.
The manuscript management system is completely online and includes a very quick and fair peer-review system, which is all easy to use. Visit http://www.dovepress.com/testimonials.php to read real quotes from published authors.

\footnotetext{
Submit your manuscript here: http://www.dovepress.com/neuropsychiatric-disease-and-treatment-journal
} 